\title{
Demencia en enfermos renales crónicos mayores de 55 años en Guatemala: prevalencia y factores asociados
}

\author{
Dementia in patients with chronic kidney disease older than \\ 55 years in Guatemala: prevalence and associated factors \\ Amarilys Alarcón-Calderón*, Carmen de Tercero, Rodrigo Aguilar, Melany Tablas, \\ Ismar López-Muralles, Emma Montejo-Camposeco. \\ Facultad de Ciencias Médicas, Universidad de San Carlos de Guatemala, Guatemala.
}

*Autor al que se dirige la correspondencia: ama.alarcon@gmail.com

Recibido: 16 de febrero 2017 / Revisión: 05 de juniio 2017 / Aceptado: 02 de octubre 2017

\section{Resumen}

$\mathrm{L}$

a demencia es un desorden que se caracteriza por un deterioro progresivo que limita la funcionalidad del individuo. Se han postulado varios factores de riesgo independientes para su desarrollo, entre ellos la enfermedad renal crónica. Se realizó un estudio transversal en 328 participantes mayores de 55 años, para determinar la prevalencia de demencia y los factores asociados en pacientes con enfermedad renal crónica. La función cognitiva de los participantes fue evaluada con la prueba cognitiva Montreal y el cuestionario de actividad funcional de Pfeffer. Se obtuvieron datos acerca de comorbilidades, valores de hemoglobina, creatinina sérica, índice de masa corporal y presión arterial. Se realizó un análisis descriptivo de la muestra, estimación de la de prevalencia de demencia y determinación de la asociación de factores de riesgo con el desarrollo de demencia por medio de regresión logística. El 16.6\% de los sujetos fueron clasificados con demencia, IC 95\% [12.82, 21.11] y 47.0\% con deterioro cognitivo leve, IC 95\% [41.54, 52.51]. Se encontró asociación positiva entre demencia y edad ( $O R$ 1.10 , IC 95\% [1.05, 1.15], $p<.001$ ), diabetes (OR 3.25, IC 95\% [1.62, 6.50], $p=.001$ ), y antecedente de trauma craneoencefálico (OR 3.28, IC 95\% [1.18, 9.09], $p=.022)$. La asociación fue negativa con hemoglobina (OR 0.71, IC 95\% [0.58, 0.88], $p=.002$ ) y tabaquismo (OR 0.31, IC 95\% [0.13, 0.78], $p=.012$ ).

Palabras claves: Disfunción cognitiva, diálisis peritoneal, diálisis renal, fallo renal crónico, función ejecutiva

\section{Abstract}

$\mathrm{D}$ ementia is a disorder characterized by progressive cognitive impairment, which limits the functionality of the affected individuals. Several independent risk factors have been postulated for its development, including chronic kidney disease. A cross-sectional design was performed in 328 subjects over 55 years old to determine the prevalence of dementia and associated risk factors in patients with chronic kidney disease. Two tests were administered to evaluate cognitive function: Montreal Cognitive Assessment and Pfeffer Functional Activities Questionnaire. Data of comorbidities, hemoglobin, serum creatinine, body mass index and blood pressure was collected. A descriptive analysis of the sample was performed, prevalence of dementia was estimated and associated factors were analyzed with a logistic regression model. $16.6 \%$ of subjects were classified as demented, whereas $47.0 \%$ had mild cognitive impairment. Significant association was found between: dementia and age (OR 1.10 CI 95\% [1.05,1.15], $\mathrm{p}<.001$ ), hemoglobin (OR .71 [.58, .88], $\mathrm{p}=.002$, diabetes (OR $3.25[1.62,6.50], \mathrm{p}=.001)$, smoking (OR .31 [.13,.78], $\mathrm{p}=.012$ ) and traumatic brain injury (OR $3.28[1.18,9.09], \mathrm{p}=.022$ ). 


\section{Introducción}

La enfermedad renal crónica (ERC) se define como una tasa de filtrado glomerular menor a $60 \mathrm{~mL} /$ min por $1.73 \mathrm{~m}^{2} \mathrm{o}$ daño renal durante tres o más meses (National Kidney Foundation, 2002). Es un problema creciente a nivel mundial, con una prevalencia estimada de hasta $13 \%$. En los últimos años, el número de enfermos renales crónicos ha ido en aumento, lo cual es una consecuencia y un reflejo del cambio de perfil epidemiológico de la población. El $20 \%$ de los pacientes con ERC terminal provienen de países en vías de desarrollo, donde se espera un aumento desproporcionado de casos (Jha et al., 2013).

En el 2013 se reconoció la ERC como un problema significativo de salud pública en Centroamérica que requiere acciones urgentes (Pan American Health Organization, 2013). En Guatemala, esta enfermedad cobra cada vez más importancia. En el 2014, la prevalencia de ERC fue de 5.4 por cada 100,000 habitantes con un incremento del $52 \%$ en comparación con la prevalencia reportada en el 2008 (Centro Nacional de Epidemiología, Ministerio de Salud Pública y Asistencia Social, 2015). Según los datos de la Unidad Nacional de Atención al Enfermo Renal Crónico (Unaerc), en ese mismo año se recibieron 1,653 pacientes nuevos (2015).

Además de los factores de riesgo tradicionales para el desarrollo de ERC (diabetes, hipertensión arterial), en Guatemala y otros países de Centroamérica se ha descrito la nefropatía mesoamericana. Esta se presenta en pacientes jóvenes agricultores de las áreas costeras, de sexo masculino. La ocurrencia de este tipo de nefropatía causa que el perfil epidemiológico de los pacientes con ERC sea distinto a otros países (Correa-Rotter, Wesseling, \& Johnson, 2014; Laux, Barnoya, Guerrero, \& Rothstein, 2015).

La evidencia epidemiológica sugiere que los pacientes con ERC tienen un mayor riesgo de desarrollar desórdenes neurológicos y demencia. Esta relación se explica en parte por las características anatómicas compartidas entre el riñón y el cerebro, así como la susceptibilidad a lesiones micro vasculares. Los pacientes con ERC tienen lesiones isquémicas subclínicas a nivel cerebral, lo que explica en parte el deterioro cognitivo (Etgen, 2015; Shen, Ruan, Yu, \& Sun, 2016). Sin embargo, la enfermedad renal crónica se asocia a demencia independientemente del daño a la microvasculatura (Yamamoto et al., 2011).

Se han propuesto hipótesis sobre los mecanismos directos en los que la ERC afecta la función y morfología cerebral, y por ende la cognición. Además, la dis- función renal provoca la acumulación de toxinas neurotóxicas (Bugnicourt, Godefroy, Chillon, Choukroun, \& Massy, 2013). La evidencia sugiere que la modalidad de tratamiento de la ERC terminal (diálisis peritoneal y hemodiálisis) puede tener consecuencias en la cognición (Lin et al., 2015; Wolfgram, Szabo, Murray, \& Whittle, 2015).

En comparación con las personas que tienen la misma edad, los pacientes que padecen de ERC, principalmente en su etapa final, tienen una prevalencia mayor de alteraciones cognitivas y una caída más rápida de sus funciones cognitivas, fenómeno que se apoya en muchos de los factores de riesgo que tienen los enfermos renales crónicos (Lin et al., 2015). La presencia de demencia ha sido asociada a una mayor tasa de complicaciones y mortalidad en esta población (Wolfgram et al., 2015).

En Guatemala, no se ha estudiado la demencia en pacientes con ERC terminal. Por lo anterior, se realizó un estudio transversal con el objetivo de estimar la prevalencia de demencia utilizando pruebas neurocognitivas y determinar factores asociados en pacientes con ERC por medio de un modelo de regresión logística.

\section{Materiales y métodos}

\section{Sujetos de estudio}

Pacientes con ERC, mayores de 55 años, con al menos dos meses de estar bajo tratamiento con hemodiálisis o con diálisis peritoneal en Unaerc, durante los meses de febrero a mayo de 2016. Los pacientes con alteración del estado de alerta o dificultades para interactuar con el medio fueron excluidos.

\section{Metodología}

Se realizó una investigación con enfoque cuantitativo de diseño transversal en una muestra de 328 participantes. La muestra fue calculada para población infinita, con una prevalencia esperada de 30 y precisión deseada de $5 \%$, con una confianza del $95 \%$. Se seleccionó a los participantes por medio de muestreo sistemático, con los listados de pacientes citados a consulta externa como marco muestral. Se registraron las características demográficas de los mismos (sexo, edad, años de escolaridad y lugar de procedencia).

Se realizó a los sujetos de estudio la Prueba Cognitiva Montreal (Tiffin-Richards et al., 2014) en su versión en español (MoCA-S) (Gil, Ruiz, Gil, Romero, \& 
Pretelt, 2015). Seguidamente se realizó el cuestionario de actividades funcionales de Pfeffer (FAQ), adaptado al español (Quiroga, Albala, \& Klaasen, 2004). Esta prueba sirve para evaluar la capacidad de los individuos para realizar actividades de la vida cotidiana, basándose en respuestas de informantes, por lo general familiares o personas que vivan con los pacientes.

Luego se procedió a la toma de presión arterial con esfigmomanómetro aneroide, y de medidas antropométricas (peso, talla, y cálculo de índice de masa corporal [IMC]) para evaluar la presencia de obesidad (IMC mayor o igual a $30 \mathrm{~kg} / \mathrm{m}^{2}$ ).

Posteriormente se revisaron los expedientes clínicos de los sujetos de estudio y se obtuvieron los datos de tipo de terapia renal sustitutiva, antecedentes de: diabetes, hipercolesterolemia, hipertensión arterial, tabaquismo, alcoholismo, trauma cráneoencefálico; valores más recientes de hemoglobina y creatinina sérica.

\section{Clasificación}

La demencia se caracteriza por deterioro en la cognición que disminuye la funcionalidad de los individuos (Asociación Americana de Psiquiatría, 2013). La cognición se evaluó con la prueba MoCA-S y la funcionalidad con el FAQ. Tomando en cuenta ambos criterios, se clasificó a los participantes tres grupos, dependiendo de los resultados obtenidos: cognición normal (ambos resultados normales), deterioro cognitivo leve (MoCA-S anormal y FAQ normal) y demencia (ambas pruebas anormales).

\section{Análisis de datos}

Se hizo un análisis descriptivo de la muestra (media y desviación estándar para las variables cuantitativas; proporciones para las variables cualitativas), con el software EpiInfo versión 7.1.5.2. Para las variables alcoholismo, diabetes, hipercolesterolemia, hipertensión arterial, obesidad, tabaquismo y antecedente de trauma craneoencefálico, se determinó su asociación como posibles factores de riesgo de demencia. Se estimaron las respectivas razones de posibilidades (odds ratio [OR]) por medio de un modelo de regresión logística, con intervalos de confianza del 95\%.

\section{Consideraciones éticas}

Inicialmente se realizó el proceso de consentimiento informado con todos los participantes del es- tudio, así como de un familiar que los acompañaba, por la vulnerabilidad de la población de estudio. El protocolo de investigación fue evaluado y aprobado por el Comité de Bioética de Investigación en Salud de la Universidad de San Carlos de Guatemala, así como por autoridades de Unaerc.

\section{Resultados}

Se obtuvieron datos de 328 participantes de los cuales $253(77.1 \%)$ recibían terapia renal sustitutiva con diálisis peritoneal y 75 (22.9\%) con hemodiálisis. De los participantes el $54.3 \%$ era de sexo femenino y el $45.7 \%$ de sexo masculino, la media (desviación estándar) de edad de los participantes fue de 63 (7.0) años y la escolaridad fue de 3.9 (3.7) años (Tabla 1). El $16.6 \%$, IC $95 \%[12.8,21.1]$ de los participantes fueron clasificados con demencia $(\mathrm{n}=54)$ y el $47.0 \%$, IC $95 \%$ $[41.5,52.5]$ como deterioro cognitivo leve $(\mathrm{n}=153)$.

Tabla 1

Características clínicas y epidemiológicas de los participantes

\begin{tabular}{ll}
\hline Característica & Estadístico \\
\hline $\begin{array}{l}\text { Edad (años) } \\
\text { Sexo }\end{array}$ & $63.4(7.0)^{\mathrm{a}}$ \\
$\quad$ Femenino & $178(54.3 \%)^{\mathrm{b}}$ \\
$\quad$ Masculino & $150(45.7 \%)^{\mathrm{b}}$ \\
Escolaridad (años) & $3.89(3.7)^{\mathrm{a}}$ \\
Modalidad de tratamiento & \\
$\quad$ Diálisis peritoneal & $253(77.1 \%)^{\mathrm{b}}$ \\
$\quad$ Hemodiálisis & $75(22.9 \%)^{\mathrm{b}}$ \\
Antecedentes & $176(53.7 \%)^{\mathrm{b}}$ \\
$\quad$ Diabetes mellitus & $99(30.2 \%)^{\mathrm{b}}$ \\
$\quad$ Alcoholismo & $80(24.4 \%)^{\mathrm{b}}$ \\
$\quad$ Tabaquismo & $79.2(14.0)^{\mathrm{a}}$ \\
Presión arterial diastólica $(\mathrm{mmHg})$ & $136.06(23.92)^{\mathrm{a}}$ \\
Presión arterial sistólica $(\mathrm{mmHg})$ & $25.02(3.77)^{\mathrm{c}}$ \\
Índice de masa corporal & $6.98(2.48)^{\mathrm{c}}$ \\
Hemoglobina (mg/dl) & $9.82(1.73)^{\mathrm{c}}$ \\
Creatinina sérica (mg/dl) & \\
&
\end{tabular}

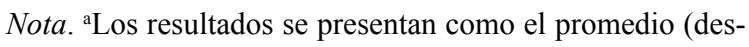
viación estándar) $(\mathrm{n}=328)$, ' Número de observaciones (porcentaje), ${ }^{\mathrm{c}}$ El promedio (desviación estándar) $(\mathrm{n}=327)$. 
El análisis ajustado mediante un modelo de regresión logística mostró asociación significativa entre demencia y edad, hemoglobina, diabetes, tabaquismo y antecedente de trauma craneoencefálico. Se encontró asociación positiva con edad, diabetes y antecedente de trauma craneoencefálico y asociación negativa con el tabaquismo y los valores de hemoglobina. No se encontró diferencia entre el grupo con modalidad de diálisis peritoneal y hemodiálisis. Al analizar por separado a los pacientes con diabetes y a los no diabéticos tampoco hubo diferencia entre ambas modalidades de tratamiento.

Las razones de posibilidades calculadas para las variables de exposición y significancia estadística, se resumen en la Tabla 2. La prueba de Hosmer-Lemeshow arrojó un valor de $11.514(p=.174)$, el valor de $-2 \log$ fue de $47.009(p<.001)$, por lo que el modelo logístico calculado es significativo.

\section{Discusión}

El deterioro cognitivo y funcional es uno de los síndromes geriátricos que ocurren con más frecuencia en pacientes con ERC que en el resto de la población (Golebiowski, Augustyniak-Bartosik, Weyde, \&
Klinger, 2016). La evidencia disponible indica que la enfermedad crónica constituye un factor de riesgo independiente para el desarrollo de alteraciones cognitivas (Etgen, Chonchol, Frstl, \& Sander, 2012).

Se ha reportado prevalencia alta de deterioro cognitivo y demencia en la población con ERC (Murray, 2008; Pereira, Weiner, Scott, \& Sarnak, 2005). Uno de los estudios pioneros en la relación de esta enfermedad con demencia mostró una prevalencia del $80 \%$ en pacientes con hemodiálisis crónica, basándose en criterios del Manual Diagnóstico y Estadístico de los Trastornos Mentales III y pruebas cognitivas (Fazekas et al., 1995). Estudios posteriores muestran prevalencia de deterioro cognitivo entre el 16 y $60 \%$ de la población con ERC (Kurella \& Yaffe, 2011; Sehgal, Grey, DeOreo, \& Whitehouse, 1997). La prevalencia de deterioro cognitivo severo se ha reportado entre el 8 y $37 \%$ (Murray, 2008; Sehgal et al., 1997).

En uno de los estudios más recientes, Foster y colaboradores (2016) reportan que los pacientes con ERC en etapas 4 y 5 presentaban deterioro cognitivo en un $61 \%$, evaluado mediante la prueba MoCa. Por otra parte, a nivel latinoamericano, se estudió recientemente en Colombia la prevalencia de deterioro cognitivo en enfermos renales crónicos en etapas 3 y 4 , con una pre-

Tabla 2

Factores de riesgo asociados a demencia, según modelo de regresión logística

\begin{tabular}{lll}
\hline Variable & OR [(IC 95\%]) & $p$ \\
\hline Edad & $1.10[1.05,1.15]$ & $<.001$ \\
Escolaridad & $0.92[0.83,-1.01]$ & .080 \\
Presión diastólica & $0.99[0.96,-1.02]$ & .512 \\
Presión sistólica & $1.01[0.99,-1.02]$ & .411 \\
Hemoglobina & $0.71[0.58,-0.88]$ & .002 \\
Creatinina sérica & $1.10[0.96,-1.26]$ & .167 \\
Tratamiento con hemodiálisis & $1.07[0.49,-2.33]$ & .871 \\
Hipercolesterolemia & $0.66[0.19,-2.29]$ & .512 \\
Diabetes & $3.25[1.62,-6.5]$ & .001 \\
Tabaquismo & $0.31[0.13,-0.78]$ & .012 \\
Alcoholismo & $1.27[0.56,-2.84]$ & .566 \\
Antecedente de trauma craneoencefálico & $3.28[1.18,-9.09]$ & .022 \\
Obesidad & $0.95[0.30,-2.97]$ & .924 \\
\hline
\end{tabular}


valencia del $51 \%$ y deterioro severo o moderado en el 15\% (Rodríguez-Angarita, Sanabria-Arenas, Diego, \& Jaramillo, 2016). En Brasil se encontró una prevalencia de deterioro cognitivo del 73.6\% (Paraizo et al., 2016), en pacientes con prediálisis.

En el presente estudio se encontraron valores de prevalencia de deterioro cognitivo similares, sin embargo, la variabilidad tan amplia entre estudios podría corresponder a distintas formas de evaluar y clasificar el deterioro cognitivo y la demencia, así como a sus diversos criterios de inclusión (algunos evalúan pacientes en etapas tempranas de la enfermedad renal crónica, mientras que otros consideran únicamente pacientes en etapa terminal, como en este estudio).

La edad constituye el factor de riesgo más importante para la demencia. Los estudios han encontrado que la incidencia de la demencia aumenta con la edad aún después de los 85 años (Larson, 2015). Como se esperaba según la evidencia disponible, el estudio identificó la edad como factor asociado a la demencia. A mayor edad, mayor probabilidad de demencia. Sin embargo, el efecto de la edad no fue tan grande como se esperaba, ya que el $O R$ fue de 1.099. Es necesario mencionar que la edad media (63.41) fue menor que en otros estudios. Si se hubiera encontrado mayor dispersión por edad, es posible que la asociación hubiera sido más fuerte.

Se ha planteado que podría existir una diferencia de riesgo de demencia según la modalidad de diálisis (hemodiálisis versus diálisis peritoneal). En el caso de los pacientes con tratamiento de diálisis peritoneal, existen datos que sugieren que la prevalencia de deterioro cognitivo es distinta a la de los pacientes con hemodiálisis (Wolcott et al., 1988; Wolfgram et al., 2015). Varios estudios sugieren que la hemodiálisis representa un mayor factor de riesgo (Tilki, Akpolat, Tunali, Kara, $\&$ Onar, 2004).

Contrario a esos hallazgos, un estudio prospectivo basado en la población no mostró un riesgo más elevado en los pacientes que reciben hemodiálisis en comparación con los pacientes en tratamiento con diálisis peritoneal (Lin et al., 2015), hallazgos similares a los encontrados en el presente estudio, en el cual no se evidenció asociación estadísticamente significativa entre la modalidad de tratamiento sustitutivo y la demencia. Sin embargo, un factor a considerar en el análisis es el tiempo en el que los pacientes han estado con una modalidad de tratamiento y si previamente habían tenido otra modalidad de tratamiento. El tiempo desde el inicio de diálisis parece ser un factor de riesgo importante para el deterioro cognitivo en los pacientes con ERC (Madero, Gul, \& Sarnak, 2008).

En concordancia con lo reportado anteriormente, este estudio mostró asociación entre diabetes y demencia. Una revisión sistemática (Biessels, Staekenborg, Brunner, Brayne, \& Scheltens, 2006) que evaluó 14 estudios longitudinales mostró que la incidencia de demencia (cualquier tipo) era más alta en pacientes con diabetes. Paraizo y colaboradores (2016) encontraron asociación entre ambas variables en pacientes enfermos renales crónicos. Los mecanismos sugeridos son alteraciones vasculares y alteraciones en el metabolismo de la glucosa, insulina y amiloide. La hipertensión arterial podría ser un mecanismo intermedio. Sin embargo, en este estudio la hipertensión no mostró asociación con la demencia.

También se encontró relación entre la demencia y antecedente de trauma craneoencefálico. La relación causal entre un único evento de trauma craneoencefálico y el riesgo de demencia no está bien establecida. La evidencia indica que los traumas leves que ocurren en personas mayores de 65 años y los traumas moderados o severos incrementan significativamente el riesgo de desarrollar demencia (Gardner et al., 2014). Sin embargo, el efecto de trauma ocurrido en edades tempranas no se conoce a ciencia cierta. El presente estudio tomó en cuenta todos los antecedentes de trauma craneoencefálico, sin importar la edad que tenía el participante al ocurrir el evento ni la severidad del trauma.

La población con ERC se encuentra propensa al desarrollo de anemia, por la falta de producción de eritropoyetina. La mayor parte de los participantes del estudio tuvieron valores bajos de hemoglobina, en rangos de anemia, con un valor medio de $9.82 \mathrm{mg} / \mathrm{dL}$. Las revisiones sistemáticas publicadas muestran una probable asociación entre la anemia y la función cognitiva, particularmente con funciones ejecutivas (Andro, Le Squere, Estivin, \& Gentric, 2013; Kurella et al., 2016; Peters et al., 2008). En concordancia con lo reportado en otros estudios, se identificó una asociación negativa de los niveles de hemoglobina con demencia. A niveles más altos de hemoglobina hay una menor probabilidad de tener demencia y viceversa.

El tabaquismo fue identificado por el modelo de regresión logística con asociación negativa con demencia. Estudios preliminares muestran información conflictiva respecto al tema. Algunos estudios muestran un efecto protector, pero los meta análisis más recientes indican que existe mayor riesgo de demencia al fumar (Anstey, von Sanden, Salim, \& O'Kearney, 
2007; Larson, 2015; van de Vorst et al., 2016; Zhong, Wang, Zhang, Guo, \& Zhao, 2015). El posible efecto protector podría explicarse por el efecto de la nicotina en los receptores nicotínicos a nivel del sistema nervioso central. Sin embargo, el tabaco tiene más efectos deletéreos que beneficiosos, entre ellos el riesgo cardiovascular. Un importante aspecto a considerar es que posiblemente los fumadores mueren antes por otras causas y no llegan a desarrollar demencia. Bajo ninguna circunstancia es válida la recomendación de fumar para prevenir la demencia.

Es necesario mencionar las limitaciones potenciales del estudio. En primer lugar, por ser un estudio de diseño transversal, no es posible establecer relaciones de causa y efecto, únicamente posibles relaciones de asociación. Se podría argumentar que algunos resultados observados son por causalidad inversa. Por último, el diagnóstico de demencia incluye un componente de progresión en el tiempo que no fue evaluado. Por lo anterior, es necesario realizar estudios prospectivos que sí permitan el análisis causal. Por otra parte, existen otras variables que no fueron tomadas en cuenta en el estudio. Por ejemplo, factores genéticos, como el alelo épsilon 4 de la apolipoproteina $\mathrm{E}$ o marcadores de inflamación, como la hiperhomocisteinemia, que la literatura identifica como factores de riesgo para la demencia (Gorelick et al., 2011; Laws, Hone, Gandy, \& Martins, 2003; Murray et al., 2006; Larson, 2015). Otro ejemplo es el antecedente de evento cerebrovascular que también se ha descrito como un factor de riesgo en este tipo de pacientes (Kavanagh, Schiller, Saxena, Thomas, \& Kurella, 2015).

Otro factor que no se consideró fue la calidad de la diálisis que estaban recibiendo los pacientes. El único indicador de uremia utilizado fue el valor de creatinina sérica, el cual no mostró asociación con la demencia. Además, no se incluyó en el análisis el tiempo desde el diagnóstico de la ERC, tiempo de tratamiento de diálisis peritoneal o hemodiálisis, ni el tiempo de presentar las comorbilidades. En este estudio solamente se incluyeron pacientes con ERC terminal, por lo que sólo se tomó en cuenta un extremo del espectro de la enfermedad.

Se puede concluir que existe una alta prevalencia de demencia y deterioro cognitivo en la población de pacientes con ERC terminal y se determinaron los factores asociados (edad, valores bajos de hemoglobina, diabetes y antecedente de trauma craneoencefálico). Sin embargo, es necesario hacer estudios prospectivos para identificar correctamente los factores de riesgo en esta población.

\section{Agradecimientos}

Esta investigación fue cofinanciada por Digi-Usac-2016, Proyecto: 4.8.63.1.84. Agradecemos a Hilda Valencia de Abril, a Federico Nave por su asesoría en el diseño metodológico y análisis estadístico de la investigación y al personal médico y administrativo de Unaerc por el apoyo brindado para la realización de la investigación, en especial a Carlos Bethancourt Monzón.

\section{Referencias}

American Psychiatric Association. (2013). Diagnostic and statistical manual of mental disorders: DSM-5 (5th. ed.). Washington, D. C.: Autor.

Andro, M., Le Squere, P., Estivin, S., \& Gentric, A. (2013). Anaemia and cognitive performances in the elderly: A systematic review. European Journal of Neurology, 20(9), 1234-1240. doi:/10.1111/ene.12175

Anstey, K. J., Von Sanden, C., Salim, A., \& O'Kearney, R. (2007). Smoking as a risk factor for dementia and cognitive decline: A meta-analysis of prospective studies. American Journal of Epidemiology, 166(4), 367-378. doi:10.1093/ aje/kwm116

Asociación Americana de Psiquiatría (2013). Manual diagnóstico y estadístico de los trastornos mentales (5a. Ed). Arlington,VA:. American Psychiatric Publishing

Biessels, G. J., Staekenborg, S., Brunner, E., Brayne, C., \& Scheltens, P. (2006). Risk of dementia in diabetes mellitus: A systematic review. Lancet Neurology, 5(1), 64-74. doi:10.1016/S14744422(05)70284-2

Bugnicourt, J.-M., Godefroy, O., Chillon, J.-M., Choukroun, G., \& Massy, Z. A. (2013). Cognitive disorders and dementia in CKD: The neglected kidney-brain axis. Journal of the American Society of Nephrology, 24(3), 353-363. doi:10.1681/ ASN.2012050536

Centro Nacional de Epidemiología, Ministerio de Salud Pública y Asistencia Social. (2015). Enfermedad renal crónica situación epidemiológica 20082015. Ciudad de Guatemala. Recuperado de http:// epidemiologia.mspas.gob.gt/files/Enfermedad_ Renal_Cronica_2015.pdf 
Correa-Rotter, R., Wesseling, C., \& Johnson, R. J. (2014). CKD of unknown origin in Central America: The case for a mesoamerican nephropathy. American Journal of Kidney Diseases, 63(3), 506-520. http://doi.org/10.1053/j.ajkd.2013.10.062

Etgen, T. (2015). Kidney disease as a determinant of cognitive decline and dementia. Alzheimer's Research \& Therapy, 7(1), 29. doi:10.1186/ s13195-015-0115-4

Etgen, T., Chonchol, M., Frstl, H., \& Sander, D. (2012). Chronic kidney disease and cognitive impairment: A systematic review and metaanalysis. American Journal of Nephrology, 35(5), 474-482. doi:10.1159/000338135

Fazekas, G., Fazekas, F., Schmidt, R., Kapeller, P., Offenbacher, H., \& Krejs, G. J. (1995). Brain MRI findings and cognitive impairment in patients undergoing chronic hemodialysis treatment. Journal of the Neurological Sciences, 134(1-2), 83-88.

Foster, R., Walker, S., Brar, R., Hiebert, B., Komenda, P., Rigatto, C., ... Tangri, N. (2016). Cognitive impairment in advanced chronic kidney disease: The Canadian Frailty Observation and Interventions Trial. American Journal of Nephrology, 44(6), 473480. doi:10.1159/000450837

Gardner, R. C., Burke, J. F., Nettiksimmons, J., Kaup, A., Barnes, D. E., \& Yaffe, K. (2014). Dementia risk after traumatic brain injury vs nonbrain trauma. JAMA Neurology, 71(12), 1490. doi:10.1001/jamaneurol.2014.2668

Gil, L., Ruiz, C., Gil, F., Romero, S. J., \& Pretelt, F. (2015). Validation of the Montreal Cognitive Assessment (MoCA) in Spanish as a screening tool for mild cognitive impairment and mild dementia in patients over 65 years old in Bogota, Colombia. International Journal of Geriatric Psychiatry, 30(6), 655-662. doi:10.1002/gps.4199

Golebiowski, T., Augustyniak-Bartosik, H., Weyde, W., \& Klinger, M. (2016). Geriatric syndromes in patients with chronic kidney disease. Postepy Higieny I Medycyny Doswiadczalnej (Online), 70(0), 581-589. doi:10.5604/17322693.1204562

Gorelick, P. B., Scuteri, A., Black, S. E., Decarli, C., Greenberg, S. M., Iadecola, C., ... Seshadri, S. (2011). Vascular contributions to cognitive impairment and dementia: A statement for healthcare professionals from the American Heart Association/ American Stroke Association. Stroke, 42(9), 26722713. doi:10.1161/STR.0b013e3182299496

Jha, V., Garcia-Garcia, G., Iseki, K., Li, Z., Naicker, S., Plattner, B., ... Yang, C. W. (2013). Chronic kidney disease: Global dimension and perspectives. Lancet, 382(9888), 260-272. doi:10.1016/S0140-6736(13)60687-X

Kavanagh, N. T., Schiller, B., Saxena, A. B., Thomas, I. C., \& Kurella, M. (2015). Prevalence and correlates of functional dependence among maintenance dialysis patients. Hemodialysis International, 19(4), 593-600. doi:10.1111/ hdi. 12286

Kurella, M., Vittinghoff, E., Yang, J., Go, A. S., Seliger, S. L., Kusek, J. W., ... Yaffe, K. (2016). Anemia and risk for cognitive decline in chronic kidney disease. BMC Nephrology, 17(1), 13. doi:10.1186/ s12882-016-0226-6

Kurella Tamura, M., \& Yaffe, K. (2011). Dementia and cognitive impairment in ESRD: diagnostic and therapeutic strategies. Kidney International, 79(1), 14-22. doi:10.1038/ki.2010.336

Larson, E. B. (2015). Risk factors for cognitive decline and dementia. En S. T. DeKosky \& J.L. Wilterdink(Eds.), UpToDate. Recuperado de https:// www.uptodate.com/contents/risk-factors-forcognitive-decline-and-dementia?source $=$ search result $\&$ search $=$ risk $\% 20$ factors $\% 20$ cognitive $\% 20$ decline\&selectedTitle $=1 \sim 150$

Laux, T. S., Barnoya, J., Guerrero, D. R., \& Rothstein, M. (2015). Dialysis enrollment patterns in Guatemala: evidence of the chronic kidney disease of non-traditional causes epidemic in Mesoamerica. BMC Nephrology, 16(1), 54. doi: 10.1186/s12882-015-0049-x

Laws, S. M., Hone, E., Gandy, S., \& Martins, R. N. (2003). Expanding the association between the APOE gene and the risk of Alzheimer's disease: Possible roles for APOE promoter polymorphisms and alterations in APOE transcription. Journal of Neurochemistry, 84(6), 1215-1236. doi:10.1046/ j.1471-4159.2003.01615.x

Lin, Y.-T., Wu, P.-H., Kuo, M.-C., Chen, C.-S., Chiu, Y.-W., Yang, Y.-H., ... Chen, H.-C. (2015). Comparison of dementia risk between end 
stage renal disease patients with hemodialysis and peritoneal dialysis - a population based study. Scientific Reports, 5, 8224. doi:10.1038/ srep08224

Madero, M., Gul, A., \& Sarnak, M. J. (2008). Cognitive function in chronic kidney disease. Seminars in Dialysis, 21(8), 29-37. doi:10.1111/j.1525139X.2007.00384.X

Murray, A. M. (2008). Cognitive impairment in the aging dialysis and chronic kidney disease populations: An occult burden. Advances in Chronic Kidney Disease, 15(2), 123-132. doi:10.1053/j.ackd.2008.01.010

Murray, A. M., Tupper, D. E., Knopman, D. S., Gilbertson, D. T., Pederson, S. L., Li, S., ... Kane, R. L. (2006). Cognitive impairment in hemodialysis patients is common. Neurology, 67(2), 216-223. doi:10.1212/01. wnl.0000225182.15532.40

National Kidney Foundation. (2002). K/DOQI Clinical Practice Guidelines for Chronic Kidney Disease: Evaluation, classification and stratification. American Journal of Kidney Diseases, 39, S1S266

Pan American Health Organization, World Health Organization (17-21 June 2013). Resolution CE152.R14 Chronic Kidney Disease in Agricultural Communities in Central America, En 152nd Session of the Executive Committee. Washington, D.C. Recuperado de http://iris.paho. org/xmlui/bitstream/handle/123456789/4786/ CE152-R14-e.pdf?sequence $=1 \&$ isAllowed $=y$

Paraizo, M. de A., Almeida, A. L., Pires, L. A., Abrita, R. S., Crivellari, M. H., Pereira, B. dos S., ... Bastos, M. G. (2016). Montreal Cognitive Assessment (MoCA) screening mild cognitive impairment in patients with chronic kidney disease (CKD) predialysis. Jornal Brasileiro de Nefrologia, 38(1), 31-41. doi:10.5935/0101-2800.20160006

Pereira, A. A., Weiner, D. E., Scott, T., \& Sarnak, M. J. (2005). Cognitive function in dialysis patients. American Journal of Kidney Diseases, 45(3), 448462. doi:10.1053/j.ajkd.2004.10.024

Peters, R., Burch, L., Warner, J., Beckett, N., Poulter, R., \& Bulpitt, C. (2008). Haemoglobin, anaemia, dementia and cognitive decline in the elderly, a systematic review. BMC Geriatrics, 8, 18. doi:10.1186/1471-2318-8-18

Quiroga, L., P., Albala B., C., \& Klaasen P., G. (2004). Validación de un test de tamizaje para el diagnóstico de demencia asociada a edad, en Chile. Revista Médica de Chile, 132(4), 467-478. doi:10.4067/S0034-98872004000400009

Rodríguez-Angarita, C. E., Sanabria-Arenas, R. M., Diego, J., \& Jaramillo, V. (2016). Cognitive impairment and depression in a population of patients with chronic kidney disease in Colombia: A prevalence study. Canadian Journal of Kidney Health and Disease, 3, 26. doi:10.1186/s40697016-0116-7

Sehgal, A. R., Grey, S. F., DeOreo, P. B., \& Whitehouse, P. J. (1997). Prevalence, recognition, and implications of mental impairment among hemodialysis patients. American Journal of Kidney Diseases, 30(1), 41-49. doi:10.1016/ S0272-6386(97)90563-1

Shen, Z., Ruan, Q., Yu, Z., \& Sun, Z. (2016). Chronic kidney disease-related physical frailty and cognitive impairment: A systemic review. Geriatrics and Gerontology International, 17(4), 529-544. doi:10.1111/ggi.12758

Tiffin-Richards, F. E., Costa, A. S., Holschbach, B., Frank, R. D., Vassiliadou, A., Krüger, T., ... Reetz, K. (2014). The Montreal Cognitive Assessment (MoCA) - A sensitive screening instrument for detecting cognitive impairment in chronic hemodialysis patients. PLoS One, 9(10), e106700. doi:10.1371/journal.pone.0106700

Tilki, H. E., Akpolat, T., Tunali, G., Kara, A., \& Onar, M. K. (2004). Effects of haemodialysis and continuous ambulatory peritoneal dialysis on P300 cognitive potentials in uraemic patients. Upsala Journal of Medical Sciences, 109(1), 4348. doi:10.3109/2000-1967-109

Unidad Nacional de Atención al Enfermo Renal Crónico. (2015). Pacientes nuevos por programa año 2016 y 2017.. Recuperado de http://unaerc. gob.gt/estadisticas/pacientes-nuevos/

van de Vorst, I. E., Koek, H. L., De Vries, R., Bots, M. L., Reitsma, J. B., \& Vaartjes, I. (2016). Effect of vascular risk factors and diseases on mortality in 
individuals with dementia: A systematic review and meta-analysis. Journal of the American Geriatrics Society, 64(1), 37-46. doi:10.1111/ jgs. 13835

Wolcott, D. L., Wellisch, D. K., Marsh, J. T., Schaeffer, J., Landsverk, J., \& Nissenson, A. R. (1988). Relationship of dialysis modality and other factors to cognitive function in chronic dialysis patients. American Journal of Kidney Diseases, 12(4), 275 284. doi:10.1016/S0272-6386(88)80220-8

Wolfgram, D. F., Szabo, A., Murray, A. M., \& Whittle, J. (2015). Risk of dementia in peritoneal dialysis patients compared with hemodialysis patients. Peritoneal Dialysis International, 35(2), 189-198. doi:10.3747/pdi.2014.00213
Yamamoto, Y., Ohara, T., Nagakane, Y., Tanaka, E., Morii, F., Koizumi, T., \& Akiguchi, I. (2011). Chronic kidney disease , 24-h blood pressure and small vessel diseases are independently associated with cognitive impairment in lacunar infarct patients. Hypertension Research, 34(12), 1276-1282. doi:10.1038/hr.2011.118

Zhong, G., Wang, Y., Zhang, Y., Guo, J. J., \& Zhao, Y. (2015). Smoking is associated with an increased risk of dementia: A meta-analysis of prospective cohort studies with investigation of potential effect modifiers. PLoS On, 10(3), 1-23. doi:10.1371/journal.pone.0118333 